\title{
Structural analysis of rat patellar tendon in response to resistance and endurance training
}

\author{
R. Barone ${ }^{1}$, M. Bellafiore ${ }^{1}$, V. Leonardi ${ }^{2}$, G. Zummo ${ }^{1}$ \\ ${ }^{1}$ Human Anatomy Section "E. Luna”, Department of Experimental Medicine, University of Palermo, 90127 Palermo, Italy, \\ ${ }^{2}$ Department of General Surgery, Emergency and Organ Transplants (GENURTO), University of Palermo, 90127 Palermo, Italy \\ Corresponding author: Rosario Barone, Human Anatomy Section "E. Luna”, Department of Experimental Medicine, \\ University of Palermo, Via del Vespro 129, 90127 Palermo, Italy. Tel: +0039 091 6553519, Fax: +0039 091 6553508, \\ E-mail: rusbarone@libero.it
}

Accepted for publication 17 July 2008

Little is known about tendon adaptations induced by mechanical loading. Our goal was to evaluate the effects of two different exercise training protocols on adult rat patellar tendon. Ninety-six male Wistar rats were divided into a sedentary group (control), a resistance-trained group and an endurance-trained group. The examinations were performed after 15, 30 and 45 days of training and after 2 weeks of rest since training was stopped. The content of collagen fibers and the cell nuclei number were quantified on tendon cross sections. In order to assess the training effectiveness, we evaluated the heart/body weight ratio, which was higher in 45 day-trained rats than their controls
$(P<0.01)$, showing the presence of cardiac hypertrophy. An increase in the content of collagen fibers was observed in the 45 day-trained groups and after 2 weeks of rest in the endurance group. Moreover, both trained groups showed a decrease in cell nuclei number after 30 and 45 days of training and 2 weeks of rest $(P<0.05)$. Endurance and resistance training induces a tendon tissue remodeling that depends on the length and intensity of workload rather than the training type. Further studies are necessary to evaluate whether these structural modifications are associated with an increase in the mechanical strength of tendon.
Tendon is mainly composed by fibrous connective tissue and connects muscle to bone. In particular, elements making up mammalian tendons are cells and extracellular matrix (ECM) including collagen fibers and ground substance (Kannus, 2000; Magnusson et al., 2003). Collagen molecules assemble into filamentous collagen fibrils, formed by microfibrils (Raspanti et al., 1990; Ottani et al., 2001) that aggregate to form collagen fibers, the main structural components (Silver et al., 2003; Provenzano \& Vanderby, 2006).

Tendon responds to mechanical loading, and animal studies have provided some evidence that endurance training may influence the morphology and mechanical properties of tendons (Viidik, 1967; Woo et al., 1980, 1981; Kubo et al., 2000; Buchanan $\&$ Marsh, 2001). Several studies have compared the biochemical composition and mechanical properties of tendons and ligaments from exercised animals with those from sedentary controls (Inglemark, 1948; Woo et al., 1980; Wren et al., 2000). In some cases, exercise training results in improved tensile strength, elastic stiffness, weight and cross-sectional area of tendons in animal experiments and their effects can be explained by an increase in collagen and ground substance synthesis by tenocytes (Woo et al., 1980; Kannus \& Natri, 1997). In other cases, exercise has no effect on these properties (Woo et al., 1981; Vailas et al., 1985). Similarly, exercise before maturity may lead to an increase in mature tendon weight (Inglemark, 1948), or it may not affect mature tendon weight (Kiiskinen, 1977; Suominen et al., 1980; Curwin et al., 1988). These inconsistencies may stem from differences in the magnitude of loading applied to various structures during general exercise programs (Tipton et al., 1986).

Only a few studies have addressed the effect of training on intratendinous structures (Davanker et al., 1996), and although one very early study has not demonstrated any intratendinous fibril increase as a result of training in rats (Inglemark, 1948), a subsequent study has shown increased fibril diameter after training in mice (Mischna, 1984; Mischna \& Hartmann, 1989).

Tendon properties and function also deteriorate with aging. The decline in muscle strength and power (Macaluso \& De Vito, 2004) is thought to be due to a loss of collagen and an increased tendon stiffness (Bailey et al., 1984). Resistance training in old age can partly reverse the deteriorating effect of aging on 
Adaptations of trained rat patellar tendon

Fig. 1. Photographs of the two methods of training. (a) Resistance training: photograph of the ladder and weight suspension on the tail of a rat. (b) Endurance training: photograph of the rota-rod treadmill.
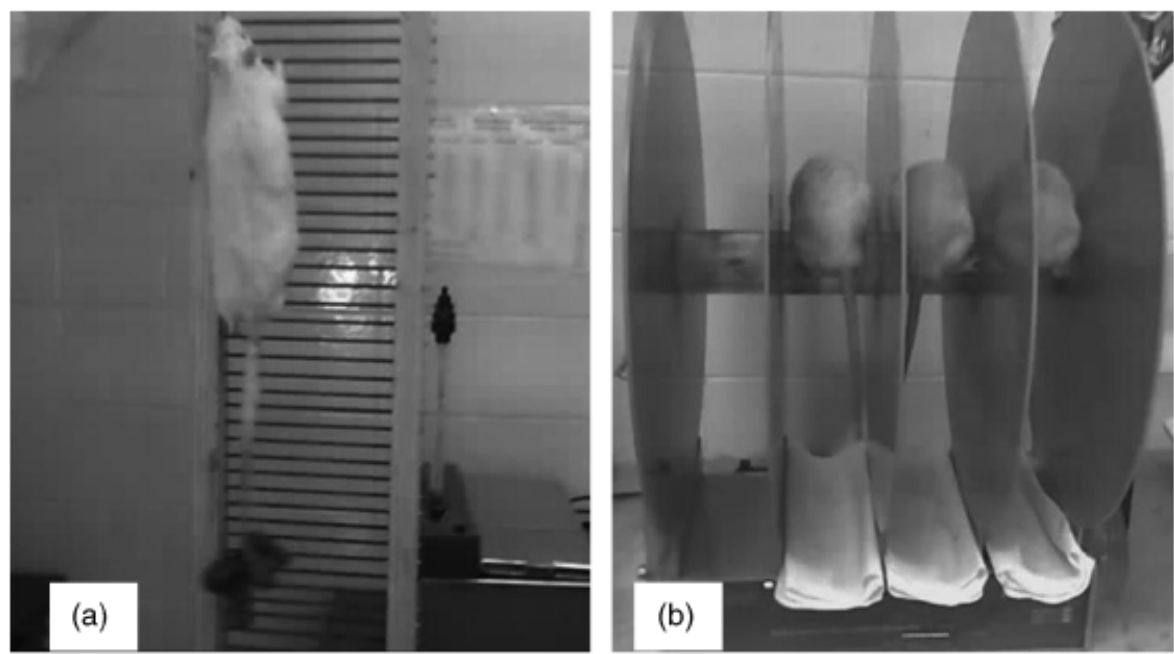

tendon properties and function (Reeves et al., 2003; Maganaris et al., 2004).

The purpose of this study was to evaluate the effects of two different training protocols (endurance and resistance) of 6 weeks' length on the structure of the patellar tendon in adult rats. In particular, we evaluated the content of collagen fibers and cell nuclei number on tendon cross sections.

\section{Materials and methods}

Experimental design

This experiment was carried out on 96 male, 6-month-old Wistar rats $(315.4 \pm 30.3 \mathrm{~g}$ body weight). The investigation conforms with the Guide for the Care and Use of Laboratory Animals (Clark, 1996). All animals were randomly divided into three groups: (1) sedentary control (C, 312.4 $\pm 25.9 \mathrm{~g}$ body weight), (2) resistance training ( $R, 319.5 \pm 27.6 \mathrm{~g}$ body weight) and (3) endurance training ( $\mathrm{E}, 314.3 \pm 35.4 \mathrm{~g}$ body weight). The sedentary group rats remained in their cages for the entire duration of the experiment. Every 2 weeks of training $(15,30$ and 45 days), eight rats from each group were weighed and then euthanized. After 45 days, the trained rats were allowed 2 weeks of rest and then they were euthanized (C60; R60; E60).

Animals were housed in cages and were allowed food and water ad libitum. The daily light cycle extended from 7 a.m. to 7 p.m. and the room temperature was maintained at $21.6 \pm$ $0.5^{\circ} \mathrm{C}$. Training was performed in the morning. We did not use unnatural incentives such as cold water, forced air or electrical stimulation in order for the rats to perform the exercise.

\section{Resistance training protocol}

An exercise consisting in climbing a $1 \mathrm{~m}$ ladder with a $2 \mathrm{~cm}$ grid ladder and weights attached to the rats' tails was used as resistance training (Fig. 1a). Rats were familiarized with the exercise for 3 days. The first week after familiarization, the exercise was performed at a natural load. In order to increase the workload, from the second week, increasing weights were attached to the base of the tail with a Velcro strap (Table 1). The initial weight was $50 \%$ of the rat body weight and gradually increased throughout the subsequent 6-week training period.
Table 1. Training protocols

\begin{tabular}{|c|c|c|c|c|c|}
\hline \multirow[t]{2}{*}{ Groups } & \multirow[t]{2}{*}{ Week } & \multicolumn{2}{|c|}{ Resistance } & \multicolumn{2}{|c|}{ Endurance } \\
\hline & & $\begin{array}{l}\text { Sets } \\
\text { (reps) }\end{array}$ & Weight (g) & $\begin{array}{l}\text { Time } \\
\text { (min) }\end{array}$ & $\begin{array}{l}\text { Speed } \\
\text { (m/min) }\end{array}$ \\
\hline \multirow[t]{2}{*}{15 Days } & $1 \mathrm{st}$ & $1(10)$ & - & 10 & 2.88 \\
\hline & 2nd & $1(10)$ & 150 & 30 & 3.60 \\
\hline \multirow{2}{*}{30 Days } & $3 r d$ & $1(10)$ & 300 & 45 & 4.32 \\
\hline & 4th & $1(10)$ & 360 & 50 & 4.32 \\
\hline \multirow{2}{*}{45 Days } & 5 th & $1(10)$ & 380 & 60 & 5.04 \\
\hline & 6 th & $1(10)$ & 380 & 60 & 5.76 \\
\hline \multirow[t]{2}{*}{60 Days } & 7th & Rest & - & Rest & - \\
\hline & 8th & Rest & - & Rest & - \\
\hline
\end{tabular}

The resistance training consisted of 1 set of 10 repetitions (reps) with a 1-min (min) rest interval among the reps, for 5 days/week (Table 1). When the rats reached the top of the ladder, they were allowed to recover in the resting area.

\section{Endurance training protocol}

A Rota-Rod Treadmill (Fig. 1b) was used for endurance training. Treadmill exercise regimes produce varying intensities of exercise by adjusting the treadmill speed and duration of exercise.

In the first week, rats ran $10 \mathrm{~min}$ at a speed of $2.88 \mathrm{~m} / \mathrm{min}$ (16laps/min) for 5 days/week and gradually increased throughout the 6-week training period (Table 1).

\section{Histological examination}

The trained and control rats were anesthetized with ether and their hearts and patellar tendons were carefully excised. The hearts were weighed on an analytical balance (LA120S Sartorius, Chicago, Illinois, USA). Patellar tendons were fixed with $10 \%$ formalin, embedded with paraffin and sectioned into serial cross sections $(6 \mu \mathrm{m})$. In order to examine the tendon in its entire length and obtain a real count of the cell nuclei number and collagen fiber content, we performed 68 cross sections for each tendon. We analyzed 20 of these sections, which had an $18 \mu \mathrm{m}$ distance from each other. Sections were stained with hematoxylin and eosin, observed with the light 


\section{Barone et al.}

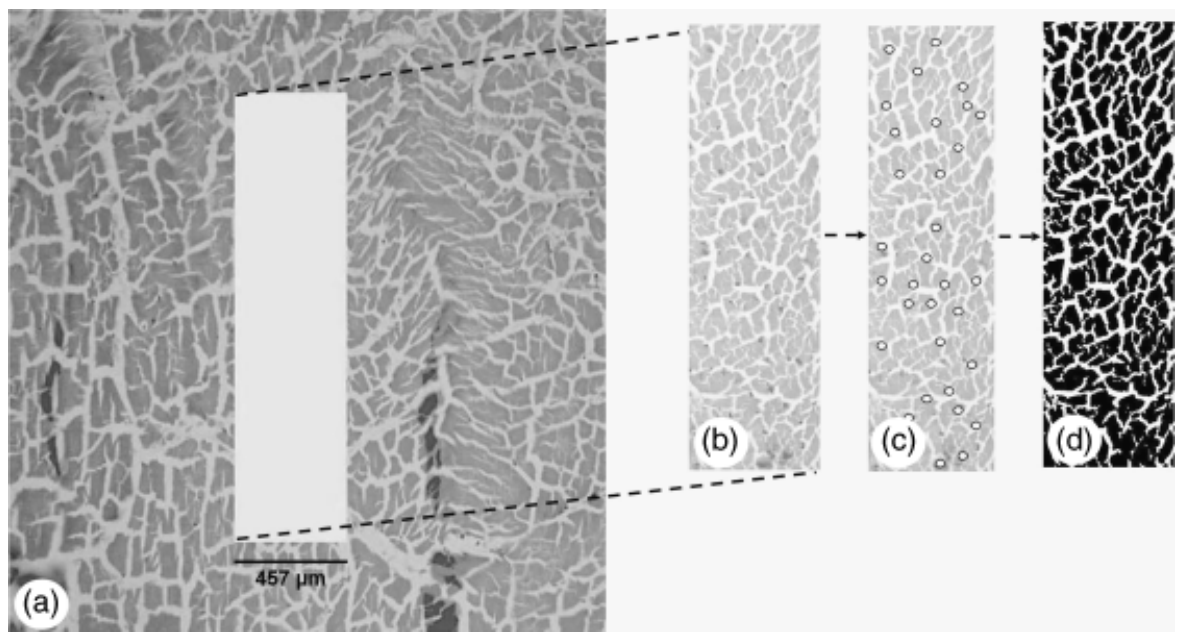

Fig. 2. Histological examination. (a) Cross sections $(6 \mu \mathrm{m})$ of patellar tendon stained with haematoxylin $\& \operatorname{eosin}(\times 20$ objective $)$; (b) area of $0.733 \mathrm{~mm}^{2}$ selected, where cell nuclei number (c) and content of collagen fibers (d) were evaluated by image software.

Table 2. Morphometric measurements from the three experimental groups

\begin{tabular}{|c|c|c|c|c|}
\hline Measurement & Control & Endurance & Resistance & $P$ \\
\hline Body weight $(\mathrm{g}) 15$ days & $340.2 \pm 08.94$ & $351.0 \pm 24.05$ & $324.7 \pm 22.10$ & - \\
\hline Body weight $(\mathrm{g}) 30$ days & $383.3 \pm 23.44$ & $308.1 \pm 12.08$ & $344.2 \pm 19.35$ & $\begin{array}{l}\text { C30 vs E30: }<0.01 \\
\text { R30 vs E30: }<0.05\end{array}$ \\
\hline Body weight $(\mathrm{g}) 45$ days & $402.1 \pm 23.66$ & $336.7 \pm 22.95$ & $399.3 \pm 54.86$ & $\begin{array}{l}\text { C45 vs E45: }<0.001 \\
\text { R45 vs E45: }<0.001\end{array}$ \\
\hline Body weight (g) 60 days & $395.7 \pm 61.53$ & $377.2 \pm 06.75$ & $383.3 \pm 18.74$ & - \\
\hline Heart weight $(\mathrm{g}) 15$ days & $1.16 \pm 0.08$ & $1.24 \pm 0.11$ & $1.13 \pm 0.07$ & - \\
\hline Heart weight $(\mathrm{g}) 30$ days & $1.37 \pm 0.03$ & $1.18 \pm 0.05$ & $1.29 \pm 0.03$ & $\begin{array}{l}\text { C30 vs R30: }<0.05 \\
\text { C30 vs E30: }<0.01 \\
\text { R30 vs E30: }<0.01\end{array}$ \\
\hline Heart weight $(\mathrm{g}) 45$ days & $1.36 \pm 0.08$ & $1.40 \pm 0.14$ & $1.68 \pm 0.17$ & $\begin{array}{l}\text { C45 vs R45: }<0.001 \\
\text { R45 vs E45: }<0.001\end{array}$ \\
\hline Heart weight $(\mathrm{g}) 60$ days & $1.35 \pm 0.17$ & $1.31 \pm 0.06$ & $1.35 \pm 0.04$ & - \\
\hline Heart /Body weight 15 days & $3.42 \pm 0.22$ & $3.62 \pm 0.21$ & $3.47 \pm 0.16$ & - \\
\hline Heart/Body weight 30 days & $3.60 \pm 0.29$ & $3.84 \pm 0.06$ & $3.76 \pm 0.30$ & - \\
\hline Heart/Body weight 45 days & $3.38 \pm 0.02$ & $4.15 \pm 0.12$ & $4.29 \pm 0.73$ & $\begin{array}{l}\text { C45 vs R45: }<0.001 \\
\text { C45 vs E45: }<0.01\end{array}$ \\
\hline Heart/Body weight 60 days & $3.41 \pm 0.13$ & $3.47 \pm 0.17$ & $3.52 \pm 0.14$ & - \\
\hline
\end{tabular}

microscopy (DM5000B Leica, Wetzlar, Germany) and the images were acquired with a digital video-camera (DC300F Leica). All images were taken with a $\times 20$ objective and a $0.733 \mathrm{~mm}^{2}$ area was selected (Fig. 2a and b), where the content of collagen fibers (Fig. 2d) and cell nuclei number (Fig. 2c) were evaluated by "Image J" (Media Cybernetics, Silver Spring, Maryland, USA) image software (Bellafiore et al., 2007).

\section{Statistical analysis}

All data are expressed as means \pm standard deviations (SD). A one-way ANOVA test with Bonferroni's multiple comparison test was used to analyze significant differences between groups and within groups. Values were considered significantly different at $P<0.05$.

\section{Results}

All of the trained rats successfully completed the 6-week training program without injury and need for any form of artificial encouragement such as electric shock incentive.

Rat body and heart weight measurements from the $\mathrm{C}, \mathrm{R}$ and $\mathrm{E}$ groups are shown in Table 2. No difference in body weight was observed between the trained groups and the $\mathrm{C}$ group after 15 days of training. The body weight of the E30 group was significantly lower than the $\mathrm{C} 30(19 \%)$ and R30 $(10 \%)$ groups $(P<0.05)$ and the body weight of E45 animals was significantly lower than the C45 $(16 \%)$ and $\mathrm{R} 45(15 \%)$ rats $(P<0.001)$. There was no difference between the trained and the $\mathrm{C}$ group after 2 weeks of rest (Table 2). A statistical analysis was also carried out within the same group. The body weight of the $\mathrm{C} 0$ group was significantly lower than the C30 (18\%), C45 (22\%) and C60 (21\%) groups $(P<0.05)$ and the body weight of the $\mathrm{C} 15$ group was significantly lower than the C30 (11\%), C45 (15\%) and C60 $(14 \%)$ groups $(P<0.05)$. The body weight of E60 animals was significantly greater than the E0 $(17 \%), \mathrm{E} 30(18 \%)$ and $\mathrm{E} 45(11 \%)$ rats $(P<0.05)$ and 
the body weight of the E30 group was significantly lower than the E15 $(12 \%)$ group $(P<0.05)$. R45 animals showed a significant increase in body weight compared with R0 (20\%), R15 (19\%) and R30 $(14 \%)$ rats $(P<0.01)$. The body weight of the R60 group was significantly greater than the R0 (17\%), $\mathrm{R} 15(15 \%)$ and R30 $(10 \%)$ groups $(P<0.05)$.

Heart weight did not show any difference between the trained groups and the $\mathrm{C}$ group after 15 days of training. The cardiac weight of the $\mathrm{C} 30$ group was significantly greater than the R30 $(6 \%)$ and E30 $(16 \%)$ groups $(P<0.05)$ and the cardiac weight of the R30 group was significantly greater than the E30 $(9 \%)$ group $(P<0.01)$. After 45 days of training there was a significantly greater difference of cardiac weight between the R45 group than the C45 (19\%) and E45 $(17 \%)$ groups $(P<0.001)$. There was no difference between the trained groups and the $\mathrm{C}$ group after 2 weeks of rest (Table 2).

The heart/body weight ratio of the $\mathrm{C} 45$ group was significantly lower than the R45 (21\%) and E45 $(19 \%)$ groups $(P<0.01)$ (Table 2$)$.

\section{Analysis of collagen fiber quantity}

The content of collagen fibers did not show any variation between the trained groups and the $\mathrm{C}$ group after 15 days of training (Fig. 3a). The content of collagen fibers of the R30 group was significantly greater than the E30 (5\%) and C30 (5\%) groups $(P<0.01)$. After 45 days of training, the $\mathrm{C} 45$ group showed a quantity significantly lower than the E45 $(4 \%)$ and R45 $(4 \%)$ groups $(P<0.05)$ (Fig. 3a). After 2 weeks of rest, the content of collagen fibers was significantly lower in the C60 group than the E60 (4\%) group $(P<0.001)$ (Fig. 3a).

A statistical analysis was also carried out within the same group in order to verify any differences in the content of collagen fibers compared with the time factor variable in the $\mathrm{C}$ group and with the time factor/training ratio in the trained groups. The content of collagen fibers was significantly greater in the C60 group than the $\mathrm{C} 15(8 \%), \mathrm{C} 30(5 \%)$ and $\mathrm{C} 45$ $(4 \%)$ control animals $(P<0.01)$. C45 rats showed significantly $(P<0.01)$ increased content collagen fibers compared with the C15 (4\%) group (Fig. 3b). The content of collagen fibers of the E60 group was significantly greater than the E15 (11\%), E30 (9\%) and E45 $(4 \%)$ animals $(P<0.001)$. E45 rats showed significantly $(P<0.01)$ increased content collagen fibers compared with E15 (7\%) and E30 (5\%) rats (Fig. 3b). Finally, the collagen fiber content of the R15 group was significantly $(P<0.001)$ lower than R30 (9\%), R45 (8\%) and R60 (10\%) animals (Fig. 3b).

\section{Evaluation of cell nuclei number}

In all the tendon cross sections stained with hematoxylin and eosin, we did not observe any presence of lymphocyte infiltration as shown in the Figs 2 and 4. No difference in cell nuclei number was observed between the trained groups and the $\mathrm{C}$ group after 15 days of training (Fig. 5a). Cell nuclei number of the C30 group was significantly greater than the E30 $(36 \%)$ and R30 (34\%) groups $(P<0.001)$ (Fig. 5a). After 45 days of training, the cell nuclei number of the C45 group was significantly greater than the E45 $(38 \%)$ and R45 groups $(42 \%)(P<0.001)$ (Figs 4 and $5 a)$. After 2 weeks of rest, cell nuclei number of the C60 group was significantly greater than the E60 $(17 \%)$ and R60 $(31 \%)$ groups $(P<0.001)$. Moreover, the E60 group showed a cell nuclei number significantly greater than the R60 $(17 \%)$ group $(P<0.001)$ (Fig. 5a).

The statistical analysis was also carried out within the same group in order to verify any differences in the cell nuclei number compared with the time factor variable in the $\mathrm{C}$ group and with the time factor/
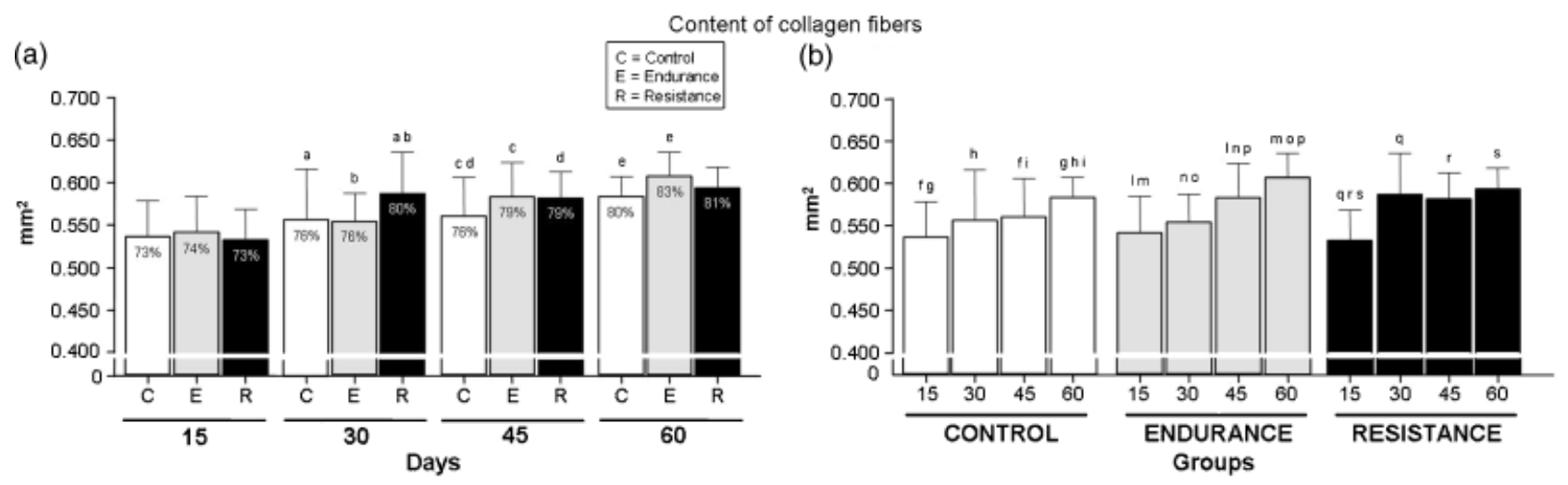

Fig. 3. Content of collagen fibers in patellar tendon sections. Column indicates means of collagen fiber content. Standard Deviations are indicated as error bars. C stands for control group; E, endurance group; R, resistance group; 15 , 15 days of training; 30, 30 days of training; 45, 45 days of training; 60, 60 days (45 days of training plus 2 weeks of rest). (a) Comparison between groups, the values in the column indicate the percentage of the collagen fiber content. (b) Comparison within group. Differences between data sets with the same lowercase letter are significant $(\mathrm{a}-\mathrm{s}): P<0.05$. 


\section{Barone et al.}

training ratio in the trained groups. The cell nuclei number of the $\mathrm{C} 15$ group was significantly lower than C30 (14\%), C45 (20\%) and C60 (14\%) animals $(P<0.001)$ (Fig. 5b). The cell nuclei number of the E15 group was significantly greater than E30 (21\%) and E45 $(16 \%)$ animals $(P<0.001)$. E60 rats showed a significant increase in cell nuclei number compared with the E30 (18\%) and E45 (17\%) groups $(P<0.001)$ (Fig. 5b). Finally, cell nuclei number of the R15 group was significantly greater than R30

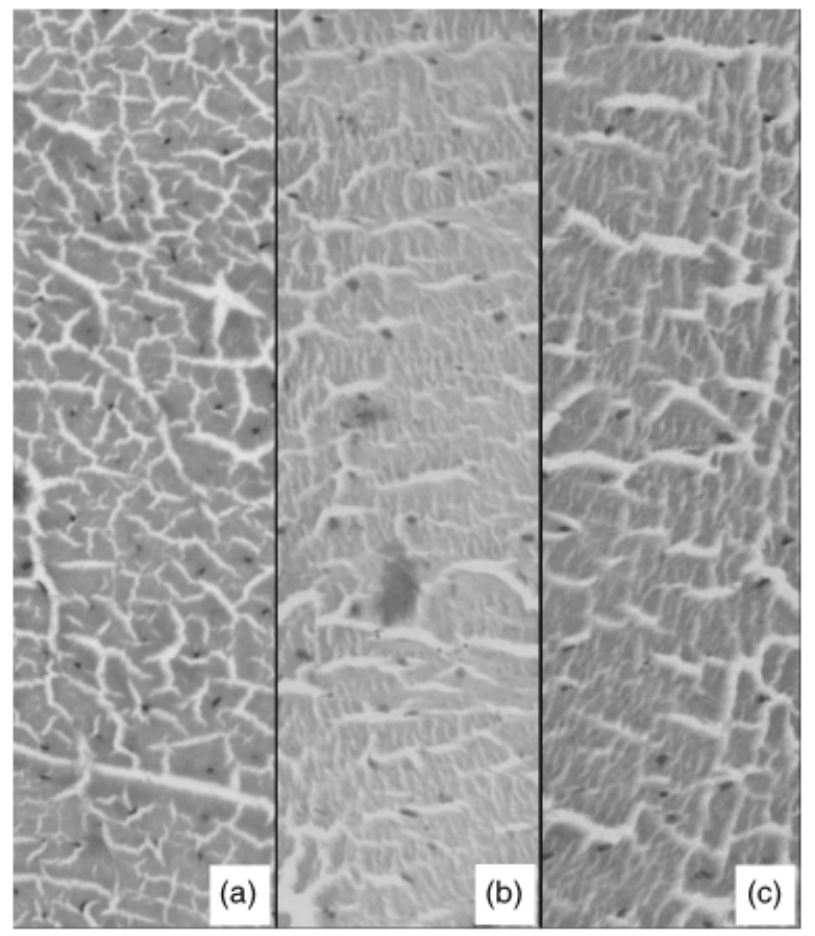

Fig. 4. Evaluation of cell nuclei number on tendon cross sections stained with hematoxylin and eosin. The images are representative of a $0.733 \mathrm{~mm}^{2}$ area from C45 (a), E45 (b) and R45 (c) groups.
$(19 \%), \quad \mathrm{R} 45 \quad(23 \%)$ and R60 (15\%) animals $(P<0.001)$ (Fig. 5b).

\section{Discussion}

Prolonged and regular exercise induces body morphological and physiological adaptations that are different depending on the intensity, duration and type of training (Curwin et al., 1988; Duncan et al., 1998).

In the present study, structural adaptations of the rat patellar tendon to different exercise training protocols were investigated. We formulated a resistance training program characterized by middleupper intensity and aimed to develop muscle mass and strength in accordance to the studies of Klitgaard (1988) and Duncan et al. (1998). The other type of exercise training (endurance protocol) was predominately aerobic, characterized by moderate intensity and rhythmic and continuous exercises. The efficiency of our endurance and resistance programs was demonstrated by several factors. The significant reduction in the body weight of E30 and E45 groups with respect to $C$ and $R$ animals might be due to the use of lipid as an energy source by aerobic metabolism during endurance training. This hypothesis is supported by increased body weight of control rats because of their sedentary life, absence of diet restriction and animal physiological growth. The increase in body weight of sedentary rats was also shown by Roy et al. (1997). The body weight of the E15 group did not significantly differ from that of R15 and C15 animals because the training intensity was lower than 30 and 45 days and glycogen deposits rather than lipids were probably used as energy sources. Although E45 rats performed higher intensity endurance training compared with E15 and E30 animals, their body weight did not show any
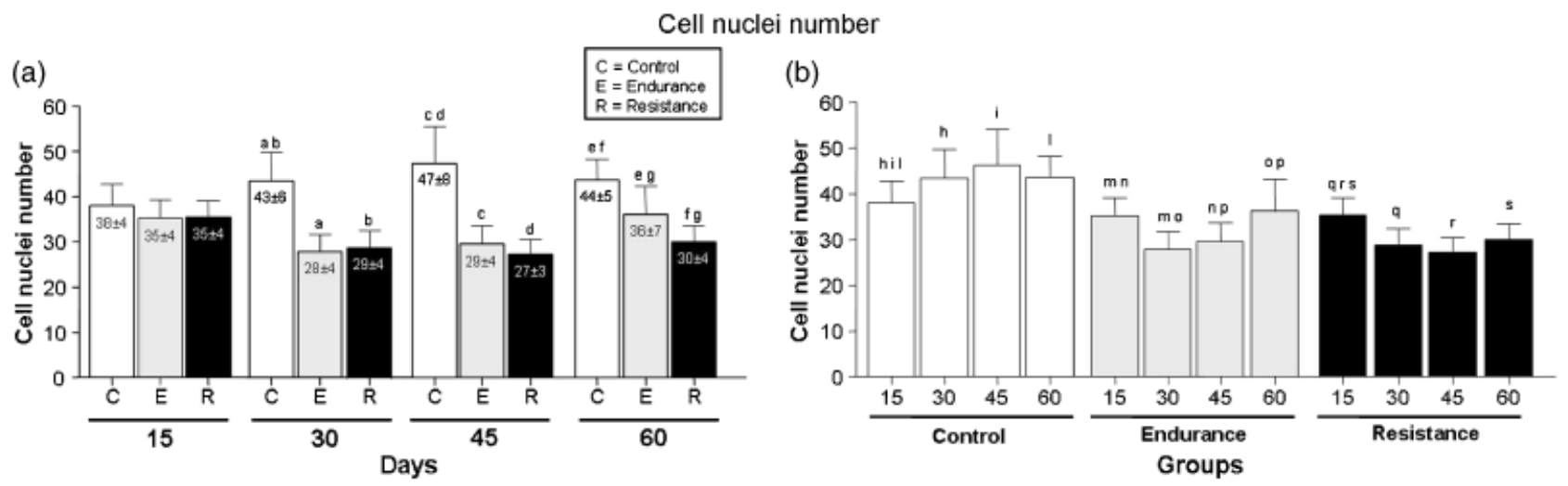

Fig. 5. Analysis cell nuclei number in patellar tendon sections. Column indicates means cell nuclei number. Standard Deviations are indicated as error bars. C, control group; E, endurance group; R, resistance group; 15, 15 days of training; 30, 30 days of training; 45, 45 days of training; 60, 60 days (45 days of training plus 2 weeks of rest). (a) Comparison between groups, the values in the column indicate the mean \pm SD of cell nuclei number. (b) Comparison within group. Differences between data sets with the same lowercase letter are significant $(\mathrm{a}-\mathrm{s}): P<0.001$. 
difference with respect to the E15 and E30 groups because the fat mass reduction of E45 rats might be compensated by an enhancement in lean mass induced by endurance training. The significant increase in E60 rat body weight compared with E0, E30 and E45 animals might be explained by the interruption of endurance training for 2 weeks.

In addition, the absence of a significant variation in the body weight of the resistance group compared with controls confirms that the decreased body weight in E30 and E45 animals is due mainly to endurance training.

The increase in the heart weight of the C30 group may be explained by an enhancement in its body weight. Indeed, R45 and C45 rats presented a similar body weight but the heart weight of the R45 group was greater than that of the control showing the efficiency of resistance training. Moreover, the significant increase in the heart/body weight ratio of endurance and resistance 45 day-trained rats compared with their corresponding controls proves the presence of cardiac hypertrophy in trained animals. An increase in absolute and relative heart mass was also observed in a study carried out by Duncan et al. (1998) on 10 male Wistar rats trained by a $40 \mathrm{~cm}$ vertical ladder while carrying progressively heavier loads secured to their tails for 26 weeks. However, the authors did not observe any difference in body mass between trained and age-matched sedentary rats (Duncan et al., 1998).

Tendon has been shown to undergo remodeling in response to exercise; however, relatively few studies have been published on the adaptations of tendon to exercise (Buchanan \& Marsh, 2002).

The response of tendon to exercise can be measured at the structural, mechanical and/or chemical level; however, most studies have been limited to measuring only one or two variables. This makes it difficult to definitively associate mechanical properties with chemical composition and structure. Several studies showed no difference in collagen concentration, respectively, in the peroneous brevis, patellar and Achilles tendons of trained rabbits, rats and chickens compared with sedentary animals (Viidik, 1967; Vailas et al., 1985; Curwin et al., 1988). Conversely, Woo et al. (1980) found that endurance training increased the collagen content of digital extensor tendons in swine; however, the same training regime had no effect on digital flexor tendons (Woo et al., 1981). The authors suggested that the different responses to exercise might be attributed to the different biochemical composition of the muscles examined (Woo et al., 1981).

In our study, the intensity and length of both training protocols stimulate the increase in tendon collagen fibers, which may be due to the mechanical load on quadriceps muscle, provoking tendon strain.
The collagen content between endurance and resistance groups changes according to the time course of the training protocol and this phenomenon may be due to motion biomechanics of posterior limbs. In particular, the different synergism between quadriceps muscle eccentric/concentric contractions performed during resistance and endurance exercise might be responsible for the different profiles in collagen concentration. Although we observed an increase in collagen fiber amount in the control group, this variation is lower than trained animals and may be due to the physiologic process of tendon growth as shown by various authors (Parry et al., 1978; Cetta et al., 1982; Nakagawa et al., 1994). Indeed, they described that the average fibril diameter and area increase during development and with age in different animal models. The enhanced content in collagen fibers might determine an improvement in tendon mechanical strength that may be relevant to carry the workload induced by exercise training in the trained groups as well as the body weight of growing control rats.

Soslowsky et al. (2000) analyzed the effects of an overuse activity on rats trained by treadmill running for 16 weeks and they found an increased cell nuclei number, tendon cells with a more rounded appearance and an altered collagen fiber organization in supraspinatus tendons. Also, Glazebrook et al. (2008) observed similar histological changes in Achilles tendon in rats trained for 12 weeks by the same protocol of Soslowsky et al. (2000). Immunohistochemical cell typing indicated that the observed increase in cellularity did not include a significant amount of inflammatory cells (leukocytes) but was mainly due to an increased number of endothelial cells and fibroblasts. Therefore, the authors suggested that these histological features could represent a biological repair/remodeling response resulting from overuse running (Glazebrook et al., 2008).

The studies of Glazebrook et al. (2008) and Soslowsky et al. (2000) showed an increase in cellularity after overuse exercise; what we observed instead is a decrease in the cell nuclei number of rat groups trained by endurance and resistance protocols. This difference may be due to the middle-upper intensity of our training protocols and the investigation methods used in our work. In particular, in order to examine the tendon in its entire length and obtain a real count, we evaluated the cell nuclei number on tendon cross sections. Conversely, Glazebrook et al. (2008) performed their experiments on tendon longitudinal sections that might not guarantee a real count of cell nuclei number because in tendon the cells are arranged in a row between the collagen fibers and, according to the kind of section cutting, cell nuclei number may considerably vary. On the other hand, cross sections performed at a 


\section{Barone et al.}

specified distance from each other can provide real counts. Until now, there are no data in the literature concerning the counting of cell nuclei number on tendon cross sections. Therefore, our work appears to be innovative because it is the first to evaluate structural modifications of tendon by a quantitative analysis and mainly in response to exercise training. This phenomenon is likely due to the difficulty in finding methods suitable for obtaining undamaged sections of tendon because of tissue hardness. Chuen et al. (2004) identified a significant number of apoptotic cells in healthy human tendon samples, in which the apoptotic index was reported to be $35 \%$ and $26 \%$ in the active remodeling sites and in tenocytes, respectively. To our knowledge, in the literature, there are no studies that have evaluated the number of apoptotic cells in tendon in response to exercise. Scott et al. (2005) showed the presence of apoptotic cells in an ex vivo rat tibialis anterior tendon model in response to short-term, high-strain mechanical loading. In our study, the decreased cell nuclei number in patellar tendons of trained rats might be a consequence of tissue remodeling induced by exercise. This hypothesis is supported by an increase in cell nuclei number after 2 weeks of rest. Moreover, this adaptation appears to be prolonged because the cell nuclei number of trained rat tendons after 2 weeks of rest continues to be lower than that of the control group.

In contrast to trained rat groups, we found a significantly greater number of cell nuclei in tendons of groups sedentary for 30, 45 and 60 days than for the 15-day control group. This datum might be explained by the increase in the dimensions of patellar tendon during growth, in agreement with Fujie et al. (2000). These authors showed the effects of stress deprivation on the dimensions and mechanical properties of the patellar tendon in 1-, 2- and 3-month-old Japanese white rabbits and found an increase in fibroblast density during growth (Fujie et al., 2000).

In conclusion, in this report we demonstrate that endurance and resistance training induces the same structural modifications in tendon tissue that appear to be rather sensitive to the length and the intensity of the workload than the training type.

\section{Perspectives}

In our study, we showed that endurance and resistance training characterized by middle-upper intensity and a length of 6 weeks causes a remodeling of tendon tissue consisting of an increase in the collagen fiber content and a reduction in the cell nuclei number. These adaptations might vary according to the animal model, motion biomechanics and tendon stress straining. Further studies are necessary to evaluate whether these structural modifications are associated with an increase in the straining strength of tendon. In the planning of training protocols, the selection of proper workloads may represent a method to provide an advantage to tendon tissue and prevent overload injuries.

Key words: running, climbing, tenocytes, exercise.

\section{Acknowledgement}

We are grateful to Dr. Marianna Zummo for the English revision of the manuscript.

\section{References}

Bailey AJ, Robins SP, Balian G. Biological significance of the intermolecular crosslinks of collagen. Nature 1984: 251: 105-109.

Bellafiore M, Sivverini G, Palumbo D, Macaluso F, Bianco A, Palma A, Farina F. Increased Cx43 and angiogenesis in exercised mouse hearts. Int J Sports Med 2007: 28: 749-755.

Buchanan CI, Marsh RL. Effects of longterm exercise on the biomechanical properties of the Achilles tendon of guinea fowl. J Appl Physiol 2001: 90: 164-171.

Buchanan CI, Marsh RL. Effects of exercise on the biomechanical, biochemical and structural properties of tendons. Comp Biochem Physiol A
Mol Integr Physiol 2002: 133: 1101-1107.

Cetta G, Tenni R, Zanaboni G, Deluca G, Ippolito E, Demartino C, Castellani AA. Biochemical and morphological modifications in rabbit Achilles tendon during maturation and ageing. Biochem $\mathbf{J}$ 1982: 204: 61-67.

Chuen FS, Chuk CY, Ping WY, Nar WW, Kim HL, Ming CK. Immunohistochemical characterization of cells in adult human patellar tendons. J Histochem Cytochem 2004: 52: 1151-1157.

Clark D. Guide for the care and use of laboratory animals. Washington, DC: National academy press, 1996.
Curwin SL, Vailas AC, Wood J. Immature tendon adaptation to strenuous exercise. J Appl Physiol 1988: 65: 2297-2301.

Davanker SP, Deane NJ, Davies AS, Firth EC, Hodge H, Parry DA. Collagen fibril diameter distribution in ligaments and tendons of the carpal region of the horse. Connect Tissue Res 1996: 34: 11-21.

Duncan ND, Williams DA, Lynch GS. Adaptations in rat skeletal muscle following long-term resistance exercise training. Eur J Appl Physiol 1998: 77: 372-378.

Fujie H, Yamamoto N, Murakami T, Hayashi K. Effects of growth on the response of the rabbit patellar tendon 


\section{Adaptations of trained rat patellar tendon}

to stress shielding: a biomechanical study. Clin Biomech 2000: 15: 370-378.

Glazebrook MA, Wright JR Jr, Langman M, Stanish WD, Lee JM. Histological analysis of Achilles tendons in an overuse rat model. J Orthop Res 2008: 26(6): 840-846.

Inglemark BE. The structure of tendons at various ages and under different functional conditions. II. An electronmicroscopic investigation from white rats. Acta Anat 1948: 6: 193-225.

Kannus P. Structure of the tendon connective tissue. Scand J Med Sci Sports 2000: 10: 312-320.

Kannus P, Natri A. Etiology and pathophysiology of tendon ruptures in sports. Scand J Med Sci Sports 1997: 7: 107-112.

Kiiskinen A. Physical training and connective tissue in young mice: physical properties of Achilles tendons and long bones. Growth 1977: 41: 123-137.

Klitgaard H. A model for quantitative strength training of hindlimb muscles of the rat. J Appl Physiol 1988: 64: 1740-1745.

Kubo K, Kaneshisa H, Kawakami Y, Fukunaga T. Elastic properties of muscle-tendon complex in longdistance runners. Eur J Appl Physiol 2000: 81: 181-187.

Macaluso A, De Vito G. Muscle strength, power and adaptations to resistance training in older people. J Appl Physiol 2004: 91: 450-472.

Maganaris CN, Narici MV, Reeves ND. In vivo human tendon mechanical properties: effect of resistance training in old age. J Musculoskelet Neuronal Interact 2004: 4: 204-208.

Magnusson SP, Hansen P, Kjaer M. Tendon properties in relation to muscular activity and physical training. Scand J Med Sci Sports 2003: 13: 211-223.

Mischna H. Morphometric analysis of loading-induced changes in collagen- fibril populations in young tendons. Cell Tissue Res 1984: 236: 465-470.

Mischna H, Hartmann G. Adaptation of tendon collagen to exercise. Int Orthopaed 1989: 13: 161-165.

Nakagawa Y, Majima T, Nagashima K. Effect of ageing on ultrastructure of slow and fast skeletal muscle tendon in rabbit Achilles tendons. Acta Physiol Scand 1994: 152: 307-313.

Ottani V, Raspanti M, Ruggeri A. Collagen structure and functional implications. Micron 2001: 32: 251-260.

Parry DA, Craig AS, Barnes GR. Tendon and ligament from the horse: an ultrastructural study of collagen fibres and elastic fibres as a function of age. Proc R Soc Lond B Biol Sci 1978: 203: 293-303.

Provenzano PP, Vanderby Jr R. Collagen fibril morphology and organization: implications for force transmission in ligament and tendon. Matrix Biol 2006: 25: 71-84.

Raspanti M, Ottani V, Ruggeri A. Subfibrillar architecture and functional properties of collagen: a comparative study in rat tendons. J Anat 1990: 172: 157-164.

Reeves ND, Narici MV, Maganaris CN. Strength training alters the viscoelastic properties of tendons in elderly humans. Muscle Nerve 2003: 28: 74-81.

Roy RR, Wilson R, Edgerton VR. Architectural and mechanical properties of the rat adductor longus: response to weight-lifting training. Anat Rec 1997: 247: 170-178.

Scott A, Khan KM, Heer J, Cook JL, Lian O, Duronio V. High strain mechanical loading rapidly induces tendon apoptosis: an ex vivo rat tibialis anterior model. Br J Sports Med 2005: 39: e25.

Silver FH, Freeman JW, Seehra GP. Collagen self-assembly and the development of tendon mechanical properties. J Biomech 2003: 36: 1529-1553.

Soslowsky LJ, Thomopoulos S, Tun S, Flanagan CL, Keefer CC, Mastaw J, Carpenter JE. Neer Award 1999. Overuse activity injures the supraspinatus tendon in an animal model: a histologic and biomechanical study. J Shoulder Elbow Surg 2000: 9: 79-84.

Suominen H, Kiiskinen A, Heikkinen E. Effects of physical training on metabolism of connective tissues in young mice. Acta Physiol Scand 1980: 108: 17-22.

Tipton CM, Vailas AC, Matthes RD. Experimental studies on the influences of physical activity on ligaments, tendons and joints: a brief review. Acta Med Scand 1986: 711: 157-168.

Vailas AC, Pedrini VA, Pedrini-Mille A, Holloszy JO. Patellar tendon matrix changes associated with aging and voluntary exercise. J Appl Physiol 1985: 58: 1572-1576.

Viidik A. The effect of training on the tensile strength of isolated rabbit tendons. Scand J Plast Reconstr Surg 1967: 1: 141-147.

Woo SL, Gomez MA, Amiel D, Ritter MA, Gelbermsan RH, Akeson WH. The effect of exercise on the biomechanical and biochemical properties of swine digital flexor tendons. J Biomech 1981: 103: 51-56.

Woo SL, Ritter MA, Amiel D, Sanders TM, Gomez MA, Kuei SC, Garfin SR, Akeson WH. The biomechanical and biochemical properties of swine tendons: long term effects of exercise on the digital extensors. Connect Tissue Res 1980: 7: 177-183.

Wren TA, Beauprè GS, Carter DR. Tendon and ligament adaptation to exercise, immobilization, and remobilization. J Rehabil Res Dev 2000: 37(2): 217-224. 